\title{
A Formal Method for Modeling Deployment Architectures Based on Bigraphs
}

\author{
Amal Gassara \\ ReDCAD, University of Sfax, B.P. 1173, 3038 Sfax, Tunisia \\ amal.gassara@redcad.org \\ ISMAEL BOUASSIDA RODRIGUEZ \\ ReDCAD, University of Sfax, B.P. 1173, 3038 Sfax, Tunisia \\ Digital Research Center of Sfax, B.P. 275, Sakiet Ezzit, 3021 Sfax, Tunisia \\ MOHAMED JMAIEL \\ ReDCAD, University of Sfax, B.P. 1173, 3038 Sfax, Tunisia \\ Digital Research Center of Sfax, B.P. 275, Sakiet Ezzit, 3021 Sfax, Tunisia
}

KHALIL DRIRA

CNRS, LAAS, 7 avenue du colonel Roche, F-31400 Toulouse, France

Univ de Toulouse, LAAS, F-31400 Toulouse, France

\begin{abstract}
Software deployment is executed according a deployment architecture which describes the allocation of software components to its hardware hosts. In this paper, we tackle the issue of constructing correct deployment architectures for large distributed systems. Actually, such architectures should satisfy various constraints related to the software components and the target environment such as the hierarchical description of components, their connections and the resource constraints. We present a formal method for constructing deployment architectures using a formal language called BRS (Bigraphical Reactive System). This method provides a correct by design approach based on multi-scale modeling ensuring the correctness of the obtained deployment architectures. Following our approach, the designer starts by modeling the first scale architecture which is refined automatically by successively adding smaller scale components until obtaining the last scale deployment architecture.
\end{abstract}

\section{INTRODUCTION}

S oftware deployment represents a sequence of related activities for placing a developed application into its target environment and making the application ready for use. For component-based applications, OMG Deployment \& Config- uration Specification (OMG D\&C Specification) [Object Management Group, 2006] outlines the following activities: installation which involves populating a repository with the application components; configuring the functionality of the installed application in the repository; planning which generates a deployment plan (i.e., describes a correct deployment archi- 
tecture); preparing the target environment by moving the application components from the repository to the specified hosts; and launching the application.

For large distributed systems, finding a correct deployment architecture is considered as a challenging task. Actually, a correct deployment architecture should respect a set of constraints related to both software components and target environment such as the hierarchy of components, their connections and their resource constraints. Satisfying these constraints make the modeling of deployment architectures more difficult.

In the literature, there are several research activities dealing with software deployment. But most of them are based on informal model and lack a solid mathematic foundation to ensure the correctness of deployment architectures. They have focused on satisfying only the resource constraints. Whereas, in our work, we propose a rigourous solution based on a formal model called BRS (Bigraphical Reactive System). Moreover, we focus on the construction of correct deployment architectures (i.e., that respect structural constraints like the hierarchy of components and their connections). Then, in our ongoing work, the efficient architecture is selected according to resource constraints.

Our method aims to help the designer to model correct deployment architectures. Instead of modeling the whole deployment architecture and verifying it with respect to defined constraint, we rather propose a correct by design approach using multi-scale modeling [Gassara et al., 2013].

Actually, in order to generate deployment architectures, we need to specify the software architecture model that describes the software components and their composition and the execution environment model that describes the target environment architecture on which application will be deployed. In fact, each model is represented as a set of scales, and each scale denotes a set of architectures.

Therefore, following our approach, the designer starts by modeling the first scale archi- tecture which is refined to give one or many architectures for the next scale. Then, these architectures are refined in turn to give the following scale architectures and so on until reaching the last scale. The transition between scales is ensured by applying specific rules which respect the defined constraints ensuring, in this way, the correctness of the obtained architectures. After constructing the architectures of both software architecture model and execution environment model, we apply the relation between the two models (i.e., integration model) in order to obtain deployment architectures.

The rest of this paper is organized as follows. In section II, research activities dealing with software deployment are presented and in section III, we present an overview of bigraphs. In section IV, we explain our bigraphical based approach for the deployment modeling. In section $\mathrm{V}$, we consider communicating systems as a study field. Then, we introduce in section VI a case study called "Smart Home" to apply our approach and its simulation with the BPL Tool in section VII. Finally, section VIII concludes this paper and gives some directions for future work.

\section{Related Work}

Various research studies have proposed methods to address the issues of software deployment. These methods include the use of OMG Deployment and Configuration $(\mathrm{D} \& \mathrm{C})$ specification [Object Management Group, 2006]. This specification offers three models. The component model defines descriptors for components and configurations, the target model defines descriptors for the target site on which applications can be deployed and the execution model defines the Deployment Plan, which describes deployment decisions. It defines an Execution Manager which executes application according to this plan.

We have identified some frameworks which have been developed on top on this specification like DAnCE [Deng et al., 2005], Dacar [Dubus and Merle, 2007] and Deploy- 
ment Factory [Hnetynka, 2005]. DAnCE is a QoS-enabled Component Deployment and Configuration Engine targeted for DRE systems. This framework deals only with CORBA Component Model. Whereas Deployment Factory is an unified environment for deploying component based applications. It proposes a generic component model which is an extension to the OMG D\&C specification. These frameworks do not provide mechanisms for redeployment and dynamic reconfiguration. However, Dacar is a model-based framework for deploying autonomic software distributed systems. It is based on a control loop and Event-Condition-Action (ECA) rules. The main limitation of these research activities is the manual deployment planning. The designer should assign the software components to the hardware ones which is a hard task especially with large scale systems.

Other research activities have proposed architecture-based approaches using ADL (Architecture Description Language) [Hoareau and Mahéo, 2006, Malek et al., 2012] and graphs [Heydarnoori and Mavaddat, 2006, Zhang et al., 2010,

Bouassida Rodriguez et al., 2008]. Hoareau et al [Hoareau and Mahéo, 2006] present a support for deploying and executing an application built with hierarchical components. It presents an ADL extension for specifying a context-aware deployment. This deployment is performed in a propagative way and is driven by constraints put on the resources of the target hosts. The framework presented in the work of Malek et al [Malek et al., 2012] aims at finding the most appropriate deployment architecture for a distributed software system with respect to multiple QoS dimensions. The framework supports formal modeling of the problem that provides a set of algorithms for finding the optimal deployment. Heydarnoori et al [Heydarnoori and Mavaddat, 2006] propose a graph based deployment planning approach for maximizing the reliability of componentbased applications. They demonstrate that this deployment problem corresponds to the multiway cut problem in graph theory. Also, the work of Zhang et al [Zhang et al., 2010] defines a component graph to represent component-based distributed applications and a tree network topology to describe the runtime environment. It defines the resource cost objective function and formulates component deployment optimization problem as mathematical programming problem.

Other research studies like [A. Dearle, 2004, Matougui and Leriche, 2012] have proposed a dedicated language (Domain Specific Language) for deployment. Dearle and Kirby [A. Dearle, 2004] propose a framework for autonomic management deployment and configuration of component-based distributed applications. An initial deployment goal is specified using Deladas (DEclarative LAnguage for Describing Autonomic Systems). A constraint solver is used to find a configuration that satisfies the goal, and the configuration is deployed automatically. If, during execution, the goal is no longer being met, a full restart of the deployment process is performed. Matougui et al [Matougui and Leriche, 2012] propose the $\mathrm{j}$-ASD middleware that addresses the autonomic deployment of ubiquitous systems. This middleware provides a DSL specifying deployment constraints. This specification is compiled into a constraint satisfaction problem, which is resolved automatically by a constraint solver. The generated deployment plan is dynamically executed by a mobile agent system.

We can note that the research activities [Hoareau and Mahéo, 2006, Malek et al., 2012, Heydarnoori and Mavaddat, 2006, Zhang et al., 2010, A. Dearle, 2004, Matougui and Leriche, 2012] deal only with resource constraints during the construction of the deployment architecture. They do not take into account the respect of structural constraints to validate he deployment architecture. Whereas, in our work, we deal with both structural and resource constraints. 


\section{i. Problem statement}

The efficiency of software systems relies on the correctness of their deployment. Actually, a deployment architecture must satisfy a set of constraints related to both software architecture (i.e, hierarchy of components and their connectivity) and target environment (i.e, structural constraints and resource constraints). Indeed, for large distributed systems with many requirements and constraints, it is hard to construct a correct deployment architecture that satisfies both structural and resource constraints manually. So, there is a need for a new method that automates the construction of the deployment architecture and guarantee its correctness. To address these issues, we propose a formal method based on the formal language Bigraph and on a multi-scale modeling approach that supports automation by a refinement process.

\section{Preliminaries}

\section{i. Bigraphs}

Bigraphs [Milner, 2001] formalise distributed systems by emphasizing both locality and connectivity. A bigraph consists principally of hyperedges and nodes which can be nested and have ports. Each hyperedge can connect many ports on different nodes (for example, $v_{0}, v_{1}$ and $v_{2}$ are joined by $e_{1}$ in Figure 1). A bigraph combines two graphical structures $-a$ place graph and a link graph-based on the same node set, hence the term bigraph.

Place graph: It is a hierarchical tree that describes the locality of the nodes. In this graph, trees are rooted by regions represented by dashed rectangles (cf. Figure 1). There can also be sites, represented as grey rectangles. A site is a hole that can host new nodes.

Link graph: It is an hypergraph that describes the connectivity of nodes. Within this graph, there can be outer names like $y_{0}, y_{1}, y_{2}$ (cf. Figure 1) and inner names like $x_{0}, x_{1}$ (cf. Figure 1) represented as open links. These names define the connection points at which

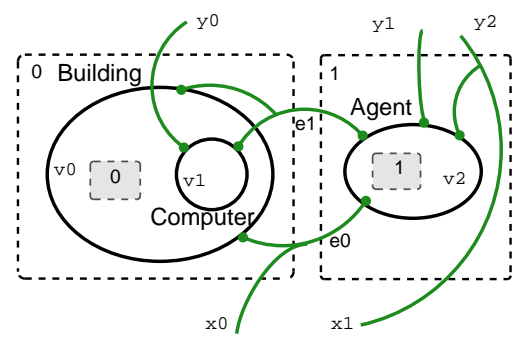

Figure 1: A Bigraph $G$

coincident names may be fused to form a single link.

Control: Each node in the bigraph is assigned a control. Controls (in the case of the bigraph G in Figure 1, Building, Computer and Agent) indicate the node type and the node ports' number through the arity. We can use the notation "X-node", which means a node that has been assigned the control $\mathrm{X}$.

Interfaces: Bigraphs can be built through their interfaces. We distinguish two types of interfaces: inner interface and outer interface. The inner interface is defined by $\mathrm{I}=<m, \mathrm{X}>$, where $m$ is the number of sites in the bigraph and $X$ the set of its inner names. The outer interface is defined by $\mathrm{J}=\langle n, \mathrm{Y}\rangle$ where $n$ is the number of regions and $Y$ is the set of outer names. In a conventional manner, the inner names are drawn below the bigraph and the outer names above it. In this example, $\mathrm{I}=<2$, $\left\{x_{0}, x_{1}\right\}>$ and $\mathrm{J}=<2,\left\{y_{0}, y_{1}, y_{2}\right\}>$.

\section{ii. Bigraphical Reactive System}

A BRS (Bigraphical Reactive System) is a set of bigraphs and a set of reaction rules that may be applied to rewrite these bigraphs. Each reaction rule consists of two bigraphs: a Redex $R$ and a Reactum $R^{\prime}$. The application of the rule consists of identifying the image of $R$ in a bigraph and replacing it by the corresponding $R^{\prime}$. For example in Figure 2, the rule allows an Agent-node to enter a Building-node which is placed in the same region. The site (grey rectangle) in the Redex represents all other possible occupants of the Building-node which are unchanged after applying this rule. 


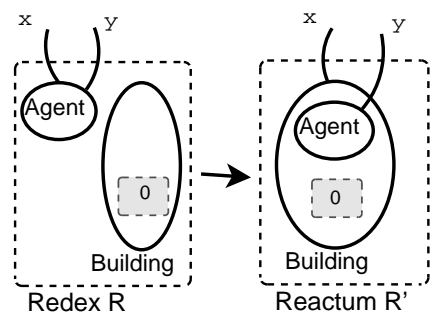

Figure 2: A reaction rule

The graphical representation used above is handy for modeling, but unwieldy for reasoning. Fortunately, bigraphs have an associated term language [Birkedal et al., 2006]. The corresponding algebraic expression (using details in

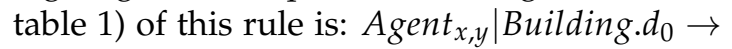
Building. $\left(\right.$ Agent $\left._{x, y} \mid d_{0}\right)$

Table 1: The term language for Bigraphs

\begin{tabular}{ll}
\hline $\begin{array}{l}\text { Algebraic } \\
\text { expression }\end{array}$ & Meaning \\
\hline $\mathrm{U}|| \mathrm{V}$ & Juxtaposition of roots \\
$\mathrm{U} \mid \mathrm{V}$ & Juxtaposition of nodes \\
$\mathrm{U} . \mathrm{V}$ & Nesting $(\mathrm{U}$ contains $\mathrm{V})$ \\
$\mathrm{K}_{x}$ & K-node linked to an outer \\
& name $x$ \\
$\mathrm{~d}_{i}$ & Site numbered $i$ \\
$/ \mathrm{xU}$ & U with outer name $\mathrm{x}$ replaced \\
& by an edge \\
\hline
\end{tabular}

\section{THE PROPOSED APPROACH}

In order to construct a deployment architecture, we need to describe the software architecture, the execution environment and the relation between them. Based on this issue, we propose an approach for deployment modeling of distributed systems which defines three models:

- Software architecture model: This model describes software components, their properties and their architecture (i.e, hierarchy of components and connections between them).
- Execution environment model: This model describes the runtime environment including physical nodes, hosts, devices, etc as well as their resource constraints and their architecture.

- Integration model: To obtain a deployment architecture, we should define the relation between the two models to map software components on physical ones.

The key objective of our work is to automate the construction of a correct deployment architecture that respects the defined models. For this, we have proposed a formal method which is based on a formal language to guarantee the correctness of the deployment architecture. This formal language should be able to describe both software and physical components. It should emphasize both hierarchy and connectivity of components. It should also provides information on both static and dynamic aspect of the system since we intend to deal with autonomic systems in future work. We have noticed that BRS is the most appropriate language that supports these requirements.

Furthermore, our formal method provides three steps to be followed as highlighted in Figure 3:

- Step 1: Description In this step, the designer describes the necessary information like software and hardware components, their properties and their resource constraints. Each component is represented with Bigraph as a node type annotated with attributes to indicate properties or available resources. The designer describes also the structural constraints through conditions on the hierarchy and the connectivity of nodes.

- Step 2: Generation In this step, the generation of the deployment architecture is performed automatically following a multiscale modeling approach. In fact, for each model (i.e., environment execution model and software architecture model), a large scale is defined by the designer. Then, it is refined by successively adding 


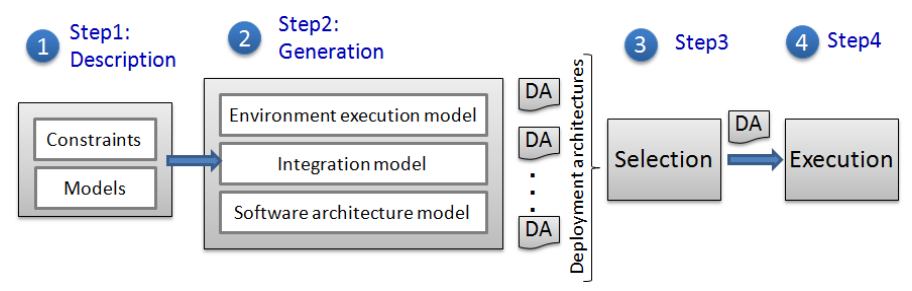

Figure 3: The proposed approach

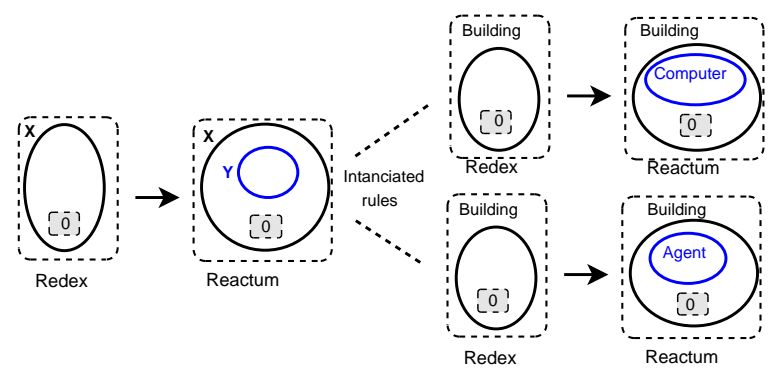

Figure 4: A meta-reaction rule for nesting a node and examples of its instantiated rules

smaller scale details until reaching the last scale. Hence, we obtain the set of possible deployment architectures by linking the two models(cf. Figure 3).

The refinement process is performed by applying specific rules. Since we aim to facilitate the modeling task for the designer, we have proposed the concept of meta-rule to describe the transition between scales. Thus, the designer identifies the corresponding meta-rule which will be instantiated automatically according to the specification in order to have the necessary rules for scale transitions. With BRS, a meta-rule is a meta-reaction rule that contains nodes having a variable control (i.e., a variable can represent any control). For example the meta-rule defined in Figure 4 allows to nest a node in another one. This meta-rule is instantiated to have two rules with the controls Building, Computer and Agent. The first one allows to nest a computer-node in a builiding-node and the second one allows to nest an agent-node in a building-node.

- Step 3: Selection In our ongoing work, a deployment architecture is selected from those generated in the previous step according to resource constraints. Hence, we obtain a deployment architecture that respects both structural and resource constraints.

- Step 4: Execution After selecting the adequate deployment architecture, it is deployed effectively using a deployment service. To do this, we intend to use the deployment service of the FACUS framework [Sancho, 2010]. This service takes as input a deployment descriptor (i.e., an XML file) and executes its by placing software components into correspondent hosts.

\section{Multi-scale MOdeling for COMMUNICATING SYSTEMS}

In our work, we address communicating systems. These systems are formed by Communicating groups. Each group is composed of devices which share common interest.

Since we aim to facilitate the modeling task to the designer, we have defined, for these systems, the scales of each model and we have defined the necessary meta-rules to be applied for the transitions between these scales and for the integration model.

\section{i. The execution environment model}

This model is represented by the following scales and transitions (we use the notation "scale $i "$ where $i$ is the scale number):

- Scale i: such $i \in[0, n]$ where $n$ corresponds to the depth of nesting in a bi- 


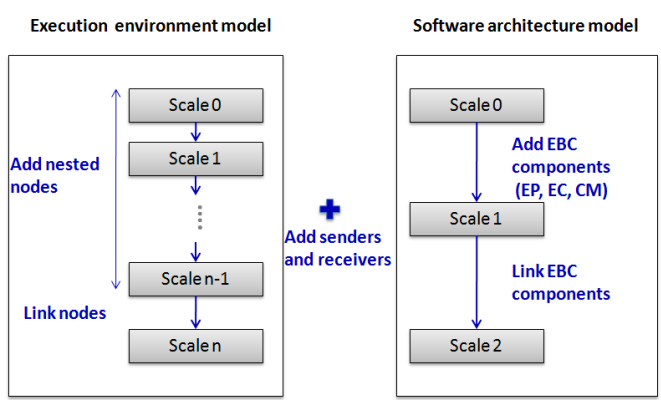

Figure 5: Multiscale modeling for communicating systems

graph. For $i=0$, we obtain the first scale.

- Transition from scale $\mathbf{i}$ to scale $\mathbf{i}+\mathbf{1}$ : The transition to the scale $i+1$ is obtained by applying a meta-reaction rule allowing to nest a node depicted in Figure 4. The corresponding algebraic expression of this rule is:

Nest a node: $X . d_{0} \rightarrow X .\left(Y \mid d_{0}\right)$

This rule enables to nest a node. So, the transition between two scales leads to increment by 1 the depth of nesting. Therefore, this meta-rule can be applied several times in order to add many nodes residing in the same node.

- Scale $\mathbf{i}+\mathbf{1}$ : such $i \in] 0, n]$. With $\mathrm{i}=n$, we obtain the scale $n$ that represents all physical entities and their composition (i.e, hierarchy). So, we reach this scale when there is no physical entities to add.

- Transition from scale $\mathbf{n}$ to scale $\mathbf{n}+\mathbf{1}$ : The transition to the scale $n+1$ is characterized by defining the link graph. So, we add hyperedges that represent the communication between different devices of the application. This operation is defined by a closure $/ x \circ G$ (i.e., outer names $x$ under a bigraph $G$ is replaced by an edge). Hence, we link nodes belonging the same communication group (i.e., having the same outer name).

- Scale $\mathbf{n}+\mathbf{1}$ : This is the last scale of the execution environment model. It represents all the physical entities and their communication.

\section{ii. The integration model}

We propose that the relation between the software architecture model and the execution environment model is a transition from scale $n+1$ of the execution environment model to scale 0 of the software architecture model. The latter includes sender and receiver components. In fact, each communication group is ensured by a set of senders and receivers. We consider that communication is done in pull mode (i.e., response to a request). So, an entity belonging a communication group should contains a pair of sender and receiver.

To ensure this transition, we define the following meta-rule:

\section{Add a sender and a receiver:}

$Y_{x} \rightarrow Y_{x} \cdot($ Sr. $x \mid R c . x)$

We nest in each node having an outername $\mathrm{x}$, a sender (Sr-node) and a receiver (Rc-node), then we nest in both of them an x-node that mark their communication group.

\section{iii. The software architecture model}

For communicating systems, the software architecture model includes the entities that take part in the communication like senders, receivers and communication middleware components. Hence, we have identified for this model the necessary components, three scales and transitions between them by defining corresponding meta-rules (cf. Figure 5).

- Scale 0: represents sender and receiver components.

- Scale 1: provides the middleware components that ensure the communication between the application components. Here, we use the Event-Based Communications (EBC) [Meier and Cahill, 2002]. EBC is a communication model which provides three types of EBC entities: event producers (EP), event consumers (EC) and channel managers (CM). The EP and EC can be connected to $\mathrm{CM}$, but they can not be directly interconnected. The EP can send data to the CM to which they are connected. The $\mathrm{CM}$ returns a copy of the received data to 
all the EC connected to it.

This scale is obtained by nesting an EPnode in each sender, an EC-node in each receiver and a CM-node for each communication group in a node that belongs to this group.

- Transition from scale 0 to scale 1: This transition is performed by applying a set of meta-reaction rules defined by the algebraic expressions given below:

Add an EP: Sr.x $\rightarrow$ Sr.EP.x

Add an EC: Rc. $x \rightarrow$ Rc.EC. $x$

Add a CM: $/ x \quad X 1_{x}\|\ldots\| X n_{x} \rightarrow / x$ $X 1_{x}\|\ldots\|\left(X n_{x} \mid C M . x\right)$

For the third rule (Add a CM), $n$ is the number of nodes belong a communication group. It will be instantiated for each communication group.

- Scale 2: This is the last scale of the software architecture model. It consists at enriching the link graph by adding new edges that link EBC components.

- Transition from scale 1 to scale 2: Reaching the scale 2 is obtained by applying a set of meta-reaction rules given below:

Link EP to CM:

EP. $x\left\|C M . x \rightarrow / y E P_{y}\right\| C M_{y} \cdot x$

Link EC to CM:

EC. $x\left\|C M . x \rightarrow / y E_{y}\right\| C M_{y} \cdot x$

\section{Case study: Smart Home}

In order to apply our approach, we consider a case study named "Smart Home" denoted in the Figure 6. Each room in a smart home can be equipped with heterogeneous devices (sensors like thermometer, presence sensor, light sensor, etc and actuators like air conditioner, lamp, etc). These devices are connected to a home gateway that manages their communication to ensure an intelligent home control like lighting control and temperature control. Sensors record information such as rooms lighting, human presence, temperature, etc. The home gateway receives these information and analyses them in order to configure the devices.
Table 2: The node controls

\begin{tabular}{lll}
\hline Control & Meaning & Arity \\
\hline $\mathrm{H}$ & Home & 0 \\
$\mathrm{R}$ & Room & 0 \\
$\mathrm{HG}$ & Home gateway & 2 \\
$\mathrm{D}$ & Device: & 1 \\
& sensor or actuator & \\
\hline
\end{tabular}

\section{i. The execution environment model}

For the smart home, the execution environment model represents home, rooms, home gateway and devices. It includes the following scales.

- Scale 0: The designer identifies the node controls as given in Table 2. Then, he models this scale using a bigraph. For the smart Home, this bigraph contains one H-node that represents a Home (cf. Figure 7).

- Transition from scale $\mathbf{0}$ to scale $\mathbf{1}$ (adding Rooms and Home Gateway): The transition to the scale 1 is obtained by instantiating the meta-rule for nesting a node. So, the rule is: $H . d_{0} \rightarrow H .\left(R \mid d_{0}\right)$.

This rule enables to add a Room (R-node) in a Home. We apply this rule as many times as the number of rooms in the home. This number is given by the designer. Here, we have 3 rooms.

The meta-rule for nesting a node is instantiated again to add a Home Gateway. The rule is: $H . d_{0} \rightarrow H$. $\left(H G \mid d_{0}\right)$

- Scale 1: This scale presents a home, three rooms and a home gateway. Its bigraph is depicted in Figure 7.

- Transition from scale 1 to scale 2 (adding Devices): The transition to the scale 2 is obtained by instantiating the meta-rule for nesting a node.

So, the rule is: $R . d_{0} \rightarrow R .\left(D \mid d_{0}\right)$.

This rule enables to add a device (D-node) in a room. We apply this rule as many times as the number of devices in the room. Here, we have 5 devices.

- Scale 2: In this scale, we obtain the bigraph specifying the home, the home 


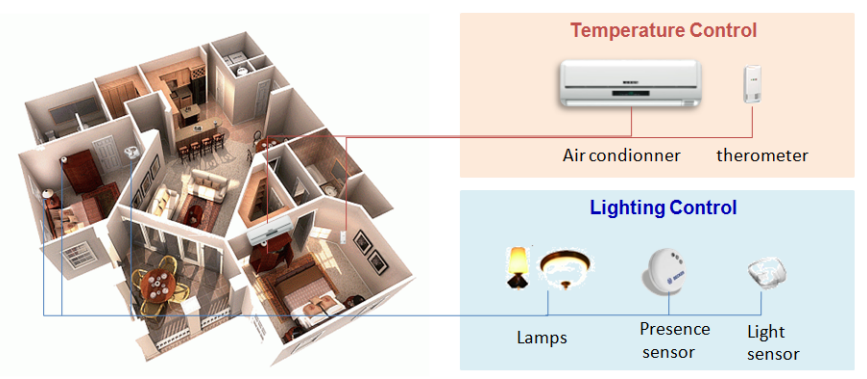

Figure 6: Smart Home

gateway and the 3 rooms. One of these rooms contains 5 devices. This bigraph is depicted in Figure 7.

- Transition from scale 2 to scale 3 (connecting entities within groups): The transition to scale 3 is obtained by applying the closure operation on the bigraph of the scale 2: / gt gloscale2

This closure operation enables to link lighting communication group (i.e., links the Home Gateway with the three devices having an outer name $g l$ : presence sensor, light sensor and lamp). It enables also to link temperature communication group (i.e., links the Home Gateway with the two other devices having an outer name $g t$ : thermometer and air conditioner).

- Scale 3: The scale bigraph is defined in the last part of Figure 7.

\section{ii. The integration model}

The transition from scale $n$ of the execution environment model to scale 0 the software architecture model is obtained by instantiating the meta-rule for adding a sender and a receiver for devices within the temperature communication group and devices within lighting communication group. For sake of shortness, we present the instantiated rules for temperature communication group:

$D_{g t} . d_{0} \rightarrow D_{g t} .\left(S r . g T|R c . g T| d_{0}\right)$

This rule enables to add a sender (Sr-node) and a receiver (Rc-node) in a device (D-node). The gT-node nested in a sender or a re-

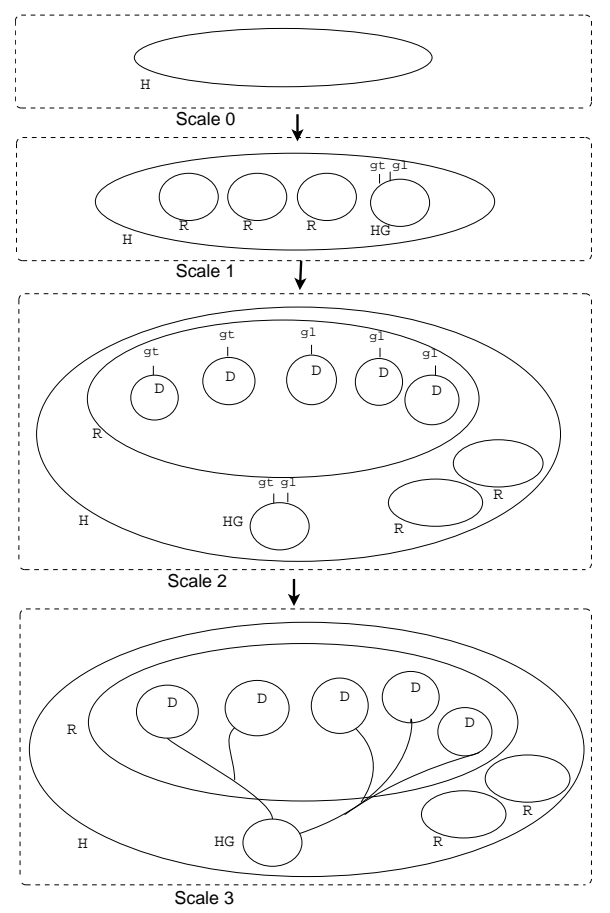

Figure 7: Scales of the execution environment model for Smart Home 
ceiver denotes the temperature communication group. We instantiate the meta-rule again to add senders and receivers in the home gateway. So the rule is:

$H G_{g t} . d_{0} \rightarrow H G_{g t} .\left(S r . g T|R c . g T| d_{0}\right)$

\section{iii. The software architecture model}

This model represents senders, receivers and EBC components.

- Scale 0: This scale represents the execution environment model including sender and receiver components.

- Transition from scale $\mathbf{0}$ to scale $\mathbf{1}$ (adding EBC components): The transition to the scale 1 is obtained by instantiating the three meta-rules of adding EP, adding EC and adding CM. So, the instantiated rules for the temperature communication group are:

Sr.gT $\rightarrow$ Sr.EP.gT

Rc.gT $\rightarrow$ Rc.Ec.gT

/gt $\quad D_{g t}|| D_{g t} \| H G_{g t} \quad \rightarrow \quad / g t$ $D_{g t}||\left(D_{g t} \mid C M . g T\right)|| H G_{g t}$

- Scale 1: At this scale, we have the execution environment model (home, home gateway, rooms and devices) with senders, receivers and EBC components. The bigraph at this scale is like the bigraph at the scale 2 depicted in Figure 8 but without the colored hyperedges. In this scale, we can obtain many Bigraphs due to the choice of the channel manager placement (i.e., the CM-node is deployed on one node belongs to the communication group).

- Transition from scale 1 to scale 2 (connecting EBC components): The transition to the scale 2 is obtained by instantiating the two meta-rules of linking an EP to a $\mathrm{CM}$ and linking an EC to a CM:

$E P . g T\left\|C M . g T \rightarrow / z E P_{z}\right\| C M_{z} \cdot g T$ $E C . g T\left\|C M . g T \rightarrow / u E C_{u}\right\| C M_{u} \cdot g T$

- Scale 2: The bigraph obtained at this scale is denoted in Figure 8. It depicts deployment infrastructure, senders, receivers and connected EBC components.

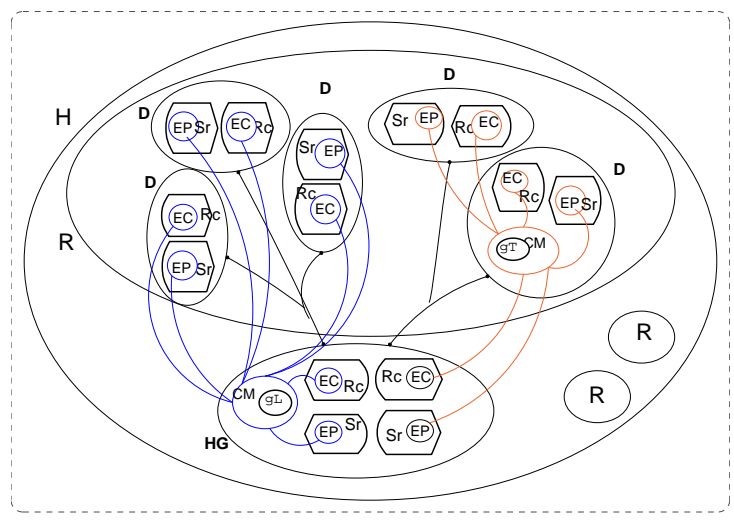

Figure 8: The Bigraph at the scale 2

So, it defines one of the set of deployment architectures.

\section{VALIDATION WITH BPL}

In order to verify the feasibility of the case study, we model our BRS using the BPL Tool (Bigraphical Programming Languages) [Hojsgaard and Glenstrup, 2011]. BPL is a tool for experimenting with bigraphical models. It provides manipulation and simulation of BRS. It relies on an SML (Standard ML) compiler with an interactive mode to provide a command line interface. The language used in the BPL Tool is called BPLL (BPL Language), and it consists of a number of SML constructs which allows to write BPLL directly in SML programs.

\section{i. Execution environment model im- plementation}

For the implementation of our case study, we create a SML file to define the BRS for the execution environment model. Listing 1 presents a portion of this file. In this listing we define:

- The signature of the system denoted in lines 2-5. It is the set of nodes controls $(\mathrm{H}$ representing the Home, $\mathrm{R}$ representing a Room, D representing a Device and HG representing the Home Gateway) 
- The rules denoted in lines 7-17 (i.e., rule for adding a room, adding a home gateway, adding a device and connecting entities within groups). For the sake of shortness, we present in listing 1 only the rules implementation that are required for the temperature communication group.

- The tactics for prescribing the sequence in which reaction rules should be applied (lines 18-22 of listing 1). According to these tactics, we apply the rule for adding a room three times, then the rule for adding a home gateway, then the rule for adding a device two times for the temperature communication group and three times for the lighting communication group and finally the connecting entities rule within the temperature communication group and within the lighting communication group.

- The initial system denoted in line 24 of listing 1. It represents the Home.

Listing 1: Execution environment model implementation

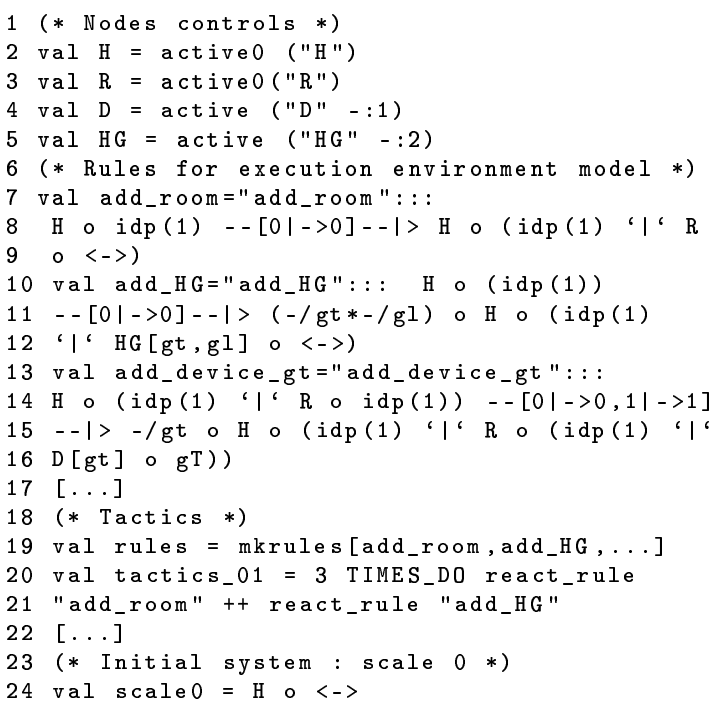

After running the simulation, we obtain the expression of each scale in BPLL. Listing 2 represents the expression of the bigraph obtained at scale 3 depicted in Figure 7. It contains the home, the rooms, the home gateway and connected devices within communication groups.
Listing 2: Scale 3 Bigraph of execution environment model

1 val scale3 $=-/ /[$ gl, gt $] \circ H \circ(\mathrm{R} \circ\langle->$ ' $\mathrm{H}$ '

$2 \mathrm{R} \circ\langle->$ '|' $\mathrm{R} \circ(\mathrm{D}[\mathrm{gt}] \circ\langle->$ '|' $\mathrm{D}[\mathrm{gt}] \circ<->$

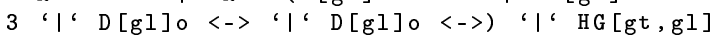
$4 \circ\langle->): 0 \quad->1$ : agent

\section{ii. Software architecture model im- plementation}

To implement the software architecture model, we complete the SML file with the nodes controls (i.e., Sr representing a Sender, Rc representing a Receiver, EC representing an Event Consumer, EP representing an Event Producer and $\mathrm{CM}$ representing a Channel Manager), the rules and their tactics (i.e., rules for adding senders and receivers, rules for adding EBC components and rules for connecting EBC components within communication groups).

After running the simulation of the software architecture model implementation, we obtain the expression of its scales in BPLL. Listing 3 denotes the bigraph obtained at scale 2 in which EBC components are connected. It corresponds to the bigraph depicted in Figure 8.

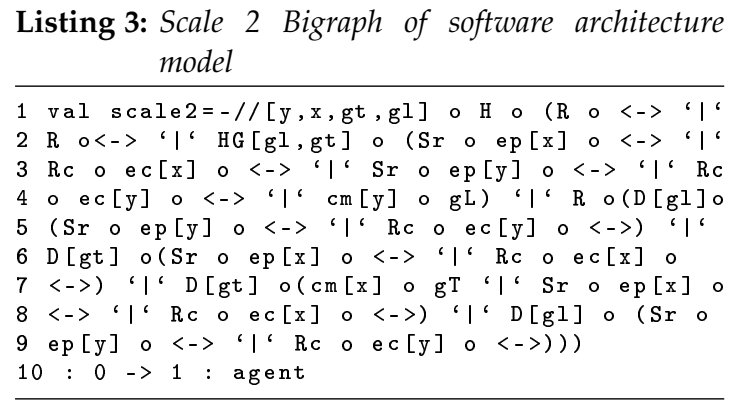

\section{CONCLUSion AND FUtURE WORK}

In this paper, we have focused on one of the challenging tasks of software deployment which consists in the construction of a correct deployment architecture. To tackle this issue, we have proposed a formal method based on bigraphical reactive systems. This formal language allows to guarantee a correct by construction architectures. This method provides 
three steps. At the first step, the designer describes the necessary information for the execution environment model, the software architecture model and the integration model. Then, the second step consists in generating automatically all the correct deployment architectures following a multiscale modeling approach. In fact, for each model, a large scale is defined by the designer. Then, it is refined by successively adding smaller scale details. This refinement process is performed by applying specific rules. Finally, the third step is the selection of the efficient deployment architecture according to resource constraints. In our work, we have addressed communicating systems. For these systems, we have identified some information in order to ease the task for the designer in the description step. In fact, we have defined the component types for the software architecture model, the scales of each model and the transition between them and also the integration model. Finally, in order to illustrate our approach, we have presented a case study called Smart Home and its implementation using BPL Tool. In future work, we aim to focus on the third step of our approach (i.e, selecting the efficient deployment architecture according to resource constraints). Then we intend to deal with autonomic systems by planning redeployment actions. Moreover, we are working at implementing a tool for Bigraph transformations since we have noticed some weaknesses of BPL Tool and we have noticed also that it does not meet our needs.

\section{REFERENCES}

[A. Dearle, 2004] A. Dearle, G. Kirby, A. M. (2004). A framework for constraint-based deployment and autonomic management of distributed applications. In International Conference on Autonomic Computing, pages 300-301.

[Birkedal et al., 2006] Birkedal, L., Debois, S., Elsborg, E., Hildebrandt, T., and Niss, H. (2006). Bigraphical models of context-aware systems. In Aceto, L. and Ingólfsdóttir, A., editors, Proceedings of the 9th International Conference on Foundations of Software Science and Computation Structure (FoSSaCS'06), volume 3921 of Lecture Notes in Computer Science, pages 187-201. Springer-Verlag.

[Bouassida Rodriguez et al., 2008] Bouassida Rodriguez, I., Van Wambeke, N., Drira, K., chassot, C., and Jmaiel, M. (2008). Multi-layer coordinated adaptation based on graph refinement for cooperative activities. Communications of SIWN, 4(1):163-167.

[Deng et al., 2005] Deng, G., Balasubramanian, J., Otte, W., Schmidt, D. C., and Gokhale, A. S. (2005). Dance: A qosenabled component deployment and configuration engine. In Component Deployment, pages $67-82$.

[Dubus and Merle, 2007] Dubus, J. and Merle, P. (2007). Towards Model-Driven Validation of Autonomic Software Systems in Open Distributed Environments. In Workshop MADAPT, in conjunction with ECOOP 2007.

[Gassara et al., 2013] Gassara, A., Bouassida Rodriguez, I., and Jmaiel, M. (2013). Towards a multi-scale modeling for architectural deployment based on bigraphs. In Software Architecture - 7th European Conference, ECSA 2013, Montpellier, France, July 1-5, 2013. Proceedings, pages 122-129.

[Heydarnoori and Mavaddat, 2006] Heydarnoori, A. and Mavaddat, F. (2006). Reliable deployment of component-based applications into distributed environments. In Proceedings of the Third International Conference on Information Technology: New Generations, ITNG'06, pages 52-57. IEEE Computer Society.

[Hnetynka, 2005] Hnetynka, P. (2005). A model-driven environment for component deployment. In Proceedings of the Third ACIS International Conference on Software Engineering Research, Management and Applications, 
SERA'05, pages 6-13. IEEE Computer Society.

[Hoareau and Mahéo, 2006] Hoareau, D. and Mahéo, Y. (2006). Constraint-based deployment of distributed components in a dynamic network. In Proceedings of the 19th international conference on Architecture of Computing Systems, pages 450-464.

[Hojsgaard and Glenstrup, 2011] Hojsgaard, E. and Glenstrup, A. J. (2011). The bpl tool: A tool for experimenting with bigraphical reactive systems. Technical Report TR-2011-145, IT University of Copenhagen.

[Malek et al., 2012] Malek, S., Medvidovic, N., and Mikic-Rakic, M. (2012). An extensible framework for improving a distributed software system's deployment architecture. Software Engineering, IEEE Transactions on, 38(1):73-100.

[Matougui and Leriche, 2012] Matougui, M. E. and Leriche, S. (2012). A middleware architecture for autonomic software deployment. In ICSNC'12: The Seventh International Conference on Systems and Networks Communications, pages 13-20. XPS.

[Meier and Cahill, 2002] Meier, R. and Cahill, V. (2002). Taxonomy of distributed eventbased programming systems. In The Computer Journal, pages 585-588.

[Milner, 2001] Milner, R. (2001). Bigraphical reactive systems: basic theory. Technical Report UCAM-CL-TR-523, University of Cambridge, Computer Laboratory.

[Object Management Group, 2006] Object Management Group, I. (2006). Deployment and configuration of component-based distributed applications specification, version 4.0.

[Sancho, 2010] Sancho, G. (2010). Adaptation d'architectures logicielles collaboratives dans les environnements ubiquitaires. Contribution à l'interopérabilité par la sémantique. Theses,
Université des Sciences Sociales - Toulouse I.

[Zhang et al., 2010] Zhang, Q., Qiu, D., Tian, Q., Sun, L., and Xu, X. (2010). Deployment planning of component-based distributed applications using mathematical programming. In Computational Intelligence and Software Engineering (CiSE 2010), pages 1 -4. 